\title{
Design of Remote Meter Reading System Based on Internet of Thing
}

\author{
Lijun Liu and Siyang Ren \\ College of Information Science and Engineering, Shenyang University of Technology Shenyang, China
}

\begin{abstract}
With the rapid development of economy, the traditional manual meter reading has been very difficult to meet the needs of social development. The demand for intelligent meter reading is growing. Remote automatic meter reading can not only replace cumbersome manual labor, but also greatly improve management effectiveness. This paper designs a kind of intelligent remote meter reading system, based on SI4432 and GPRS. The system is composed of electric energy meter, data collector and management software. The data collector includes a power module, a sampling module and a communication module. The software includes a database query module, a real-time meter reading module, and finally displays and counts various information data in the form of a web page so as to operate on the Internet. The system has the characteristics of low power consumption, low cost and intelligence, so that it can be used widely.
\end{abstract}

Keywords—remote meter reading; SI4432; GPRS

\section{INTRODUCTION}

In the field of power system, the rapid development of smart grid brought a new revolution to the life of people, while the world demand for energy is also increasing in, the remote automatic meter reading demand also will increase ${ }^{[1]}$. Because the remote automatic meter reading can access the data information related to users' electricity in real time, it not only brings convenience to power department's statistical power consumption, but also provides guarantee for accident prevention. Remote automatic intelligent meter reading system is safe and convenient technology to power supply department, it can save a lot of manpower material resources, also can better adapt to the complex environment, at the same time for the gas and water and other public service sector meter reading method to provide some reference.

\section{SySTEM OVERALL STRUCTURE DESIGN AND HARDWARE PLATFORM CONSTRUCTION}

\section{A. System Overall Design Scheme}

The remote meter reading system is mainly composed of the wireless meter, database, centralized controller and monitor display interface. The overall structure of the system is shown in figure I.

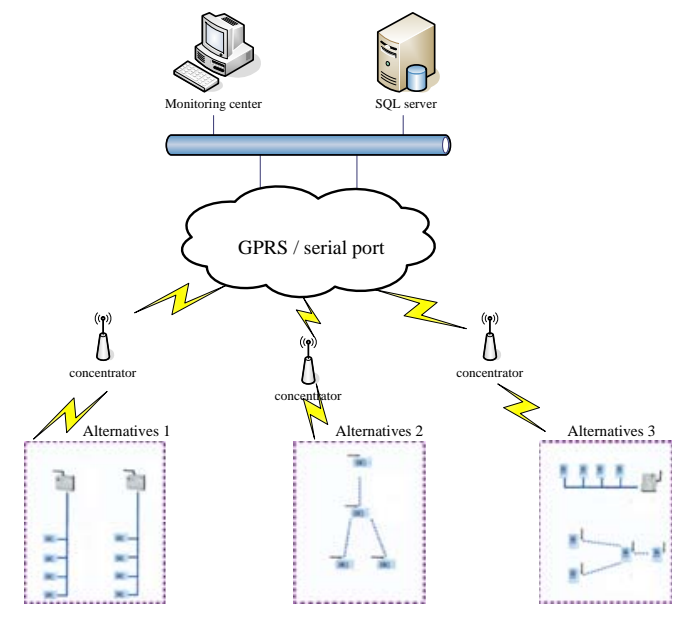

FIGURE I. OVERALL STRUCTURE OF SYSTEM

There are three kinds of system schemes, of which the second scheme adopts wireless transmission mode, which has unmatched advantages. This topic carries on the research and the design to this scheme. The system is based on WIAPA technology standard and uses SI4432 chip as a wireless transmission module. GPRS is used to realize long-range communication. After the power meter detects the power information, it is transmitted to the concentrator through the wireless transmission system. The concentrator then uses GPRS to connect to the server and writes the data Into the SQL server database. When the user accesses the meter information through the Internet, the system will display the detected information in the form of a webpage. When the user wants to view the historical information, he can click the corresponding button on the web page to view the historical data so as to realize the intelligent management. The theoretical value of the WIAPA module support node is about 200. Multiple modules can be used to carry more nodes. In this paper, a module Design as a basis for introducing system design.

\section{B. Hardware Platform Construction}

According to the overall scheme and transmission distance of the above system, STM32F103 is adopted as the hardware platform microprocessor, SI4432 as a wireless transceiver module to form a wireless LAN hardware platform, which Si4432 is Silicon Company, using 433MHZ wireless communications, a high-performance chip. It has the advantages of low frequency, long wavelength, low transmission rate, strong diffraction ability and long communication distance ${ }^{[2]}$. 


\section{1) Design of energy meter}

Energy meter node structure block diagram is shown in figure 2.The system uses a smart meter to measure electricity consumption. The processor collects electricity and stores processing. It is sent to the upper computer through the wireless transceiver module.

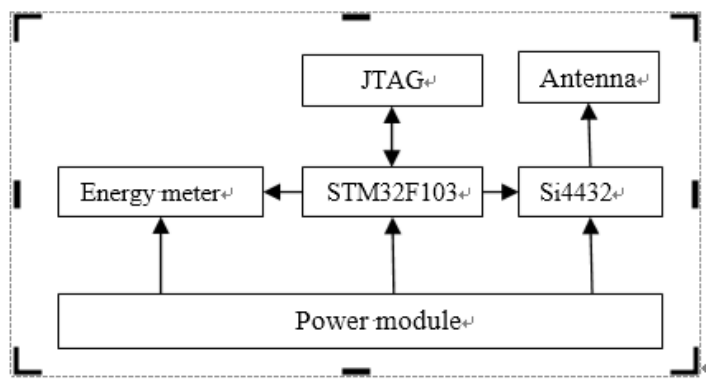

FIGURE II. ENERGY METER NODE STRUCTURE BLOCK DIAGRAM

In the system, the collection node module needs to collect and manage the data in real time or in time. The data are stored and managed and transmitted through SI4432.The wireless transmission module of this system uses the energy meter for power supply, which belongs to the low power consumption module. The STM32F103 peripheral circuit and the peripheral circuit diagram of SI4432 are given, in Figure 3 and figure 4.

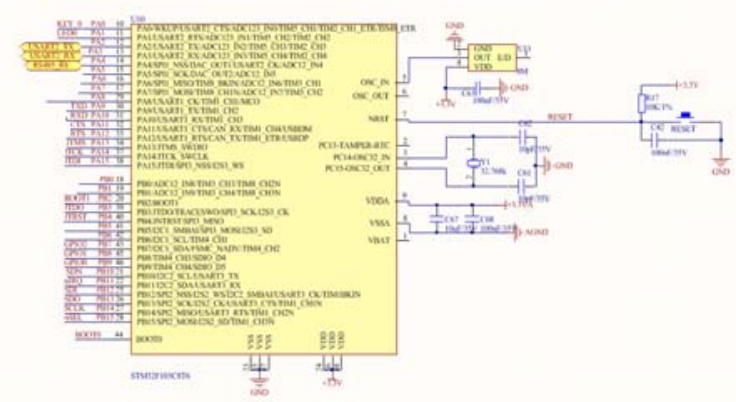

FIGURE III. PERIPHERAL HARDWARE CIRCUIT OF STM32

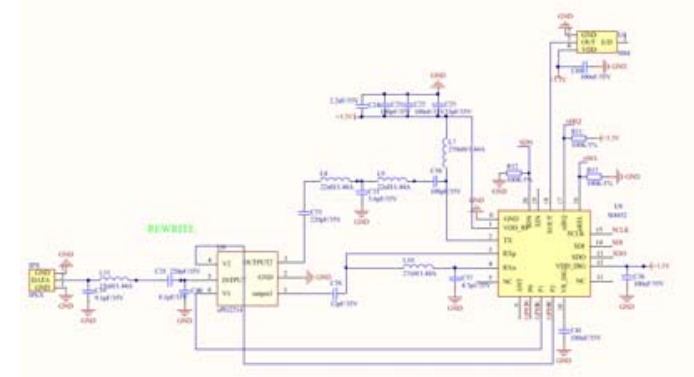

FIGURE IV. PREIPHERAL HARDWARE CIRCUIT OF SI4432

2) Design of centralized controller and remote transmission communication

The centralized controller mainly includes the power supply part, the wireless transmission module, STC12C5A60S2, and the GPRS. Its main function is to receive the data of the energy meter nodes and upload them to the remote host via the GPRS module. Block diagram of centralized controller is shown in figure 5.

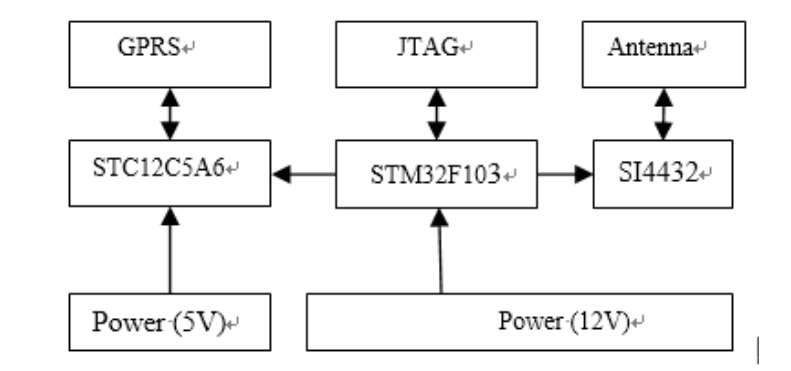
FIGURE V. CENTRALIZED CONTROLLER STRUCTURE BLOCK
DIAGRAM

The STC12C5A60S2 chip is chosen as the main control chip of the centralized controller, it is the single-clock machine cycle (1T) microcomputer produced by STC company, the peripheral circuit is shown in figure 6 .
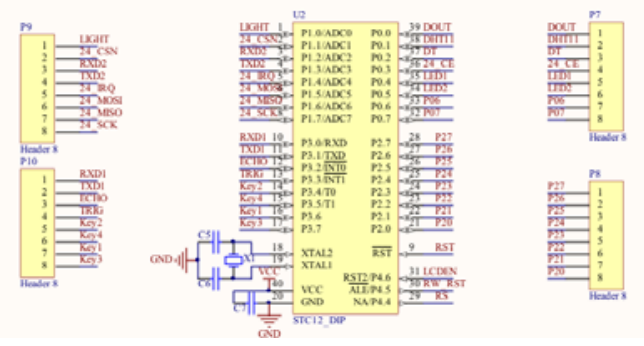

FIGURE VI. PERIPHERAL HARDWARE CIRCUIT OF STC12C5A6

The concentrator sends the server meter reading instructions to the collector, and the MCU unit in the collector processes and interprets the meter reading commands. The data of the connected tables under the collector channel are promptly uploaded to the concentrator through the Si4432 wireless module. SMS, data and fax information with low power consumption. GPRS communication module combines GPRS network technology to realize the communication between monitoring data and upper computer through Internet network [6]. The serial communication circuit of STC12C5A60S2 and SIM900A and CC2530 is shown in figure 6.The communication between STC12C5A60S2 and the coordinator and SIM900A is realized through serial port.

\section{SYSTEM SOFTWARE DESIGN}

\section{A. Wireless LAN Software Design}

A wireless LAN is a star network consisting of a coordinator and multiple terminal nodes. The communication from the node to the coordinator is in the form of one to one unicast. From the coordinator to the node communication in the form of broadcasting, and each terminal node judges the device receiving the command by matching the cluster ID.[4]

The software design of wireless LAN is mainly divided into the design of the collector software and the design of the concentrator software. 
The main work of the collector node software design is the hardware initialization, data acquisition and processing, and the wireless transmission of the concentrator. When the master Si4432 and slave station to establish a connection, the concentrator sends the server meter reading instructions to the collector, and the MCU unit in the collector processes and interprets the meter reading commands. The data of the connected tables under the collector channel are promptly uploaded to the concentrator through the Si4432 wireless module.

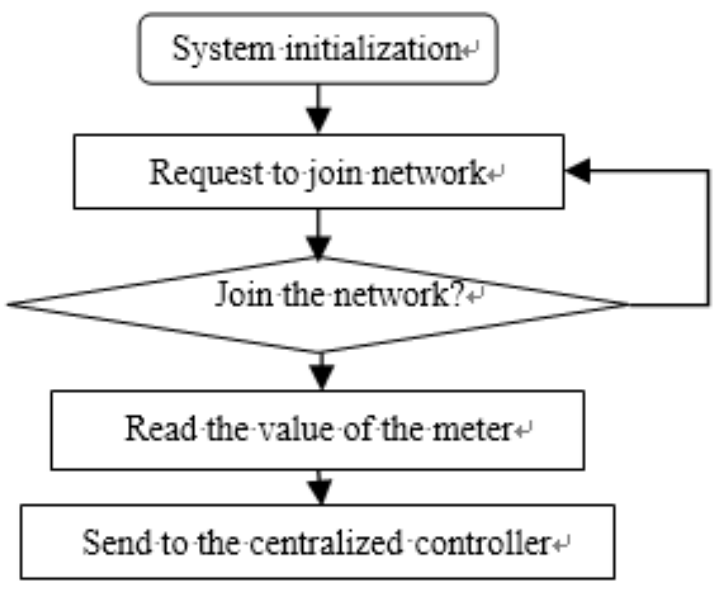

\section{FIGURE VII. ENERGY METER NODE DESIGN FLOW CHART}

The main work of the concentrator software design is the initialization of the hardware, the establishment of the network, the wireless communication with the power meter node and the serial communication with the controller. The concentrator, as a bridge between the collector and the server, plays an important role in the whole system. GPRS is the medium of the communication between the server and the concentrator. The server sends instructions through the GPRS to the concentrator, and the MCU handles the upper computer. When the concentrator starts to work, firstly, the GPRS module is powered up to register the network, then set the network configuration parameters, open the Internet service, and establish a network connection with the host computer [5].When the GPRS is online, the MCU handles the command to control Si4432 to set up a connection. When GPRS is not logged in to the server or GPRS is disconnected for some reason, the system will automatically detect the unregistered state, restart GPRS and start the network connection with the server.

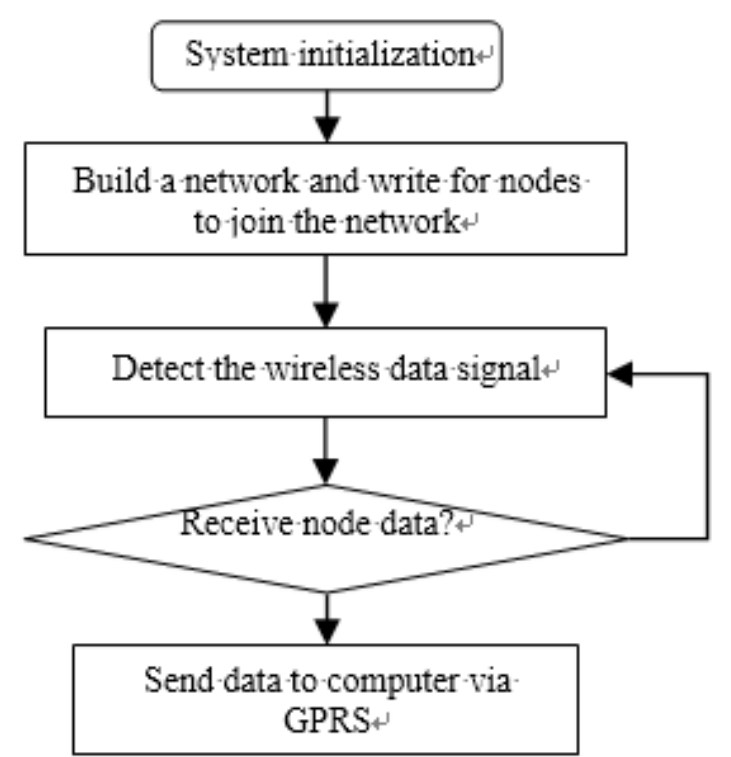

\section{FIGURE VIII. CENTRALIZED CONTROLLER DESIGN FLOW CHART}

\section{B. Monitoring Center Software Design}

The main function of the upper computer is to set up a good man-machine interface. It is convenient for the meter reading clerk to send the meter reading commands, set the concentrator and collector parameters remotely, store and process the meter reading data, analyze the statistics, inquire the electricity and operate the state of the electric energy meter under the remote monitoring system. PC interface is divided into the landing module, the query module, the remote automatic meter reading management module and the management module. The function modules of the upper computer cooperate with each other so as to ensure the normal operation of the whole system.

The design of the GPRS to send data to the monitoring center is completed through the network communication based on SOCKET, the client to the server sends service request and by the typical mode of the server after receiving the corresponding service, namely the client / server model (Server/Client), can be used in the above communication between different hosts in the network ${ }^{[6]}$.

\section{SySTEM TEST}

This paper has carried out two aspects of testing. First, wireless LAN quality testing; Second, remote transmission and communication testing.

\section{A. Wireless LAN Quality Test}

In the IEEE802.15.4 protocol, we use the packet loss rate to test the connection quality. This paper uses second kinds of test methods, test sites for lab building, In the open corridor, the obstacle-free experiment environment is simulated, and obstacle experiments are performed in the laboratory. In each experiment environment, the nodes are respectively placed at 
positions of $0.5 \mathrm{~m}$ and $1 \mathrm{~m}$ from the ground, and the antenna of the node is perpendicular to the ground.

The total amount of a single test packet is set to 1000, and the packet transmission speed is10/s. To verify the relationship between the packet loss rate and the node distance, the distance between nodes is determined to be $0.2,0.5,10,20,60$, $100120 \mathrm{~m}$. Test results shown in Table 1 :

TABLE I. The RELATIONSHIP BETWEen PACKet LosS RATE AND COMMUNICATION DISTANCE

\begin{tabular}{|l|c|c|c|}
\hline Distance(m) & Sending Packets & $\begin{array}{c}\text { Receiving } \\
\text { Packets }\end{array}$ & $\begin{array}{c}\text { Packet } \\
\text { Loss(\%) }\end{array}$ \\
\hline 0.2 & 1000 & 1000 & 100 \\
\hline 0.5 & 1000 & 1000 & 100 \\
\hline 10 & 1000 & 1000 & 100 \\
\hline 20 & 1000 & 1000 & 100 \\
\hline 60 & 1000 & 997 & 99.7 \\
\hline 100 & 1000 & 1000 & 100 \\
\hline 120 & 1000 & 1000 & 100 \\
\hline
\end{tabular}

The analysis data can be found in the near range (20m), the data packet loss rate is very low, basically no packet loss. A data packet loss situation appeared near $60 \mathrm{~m}$. Through the test, within $120 \mathrm{~m}$, even in the case of the maximum packet loss rate, the effective receiving rate of the packet is also $99.7 \%$, which meets the design requirements.

\section{B. Remote Transmission Communication}

System parameter configuration interface as shown. Click the system configuration, the system configuration interface, set the experimental baud rate to $9600 \mathrm{bps}$, set the machine ip and port number. Stop bit 1, parity is even parity.

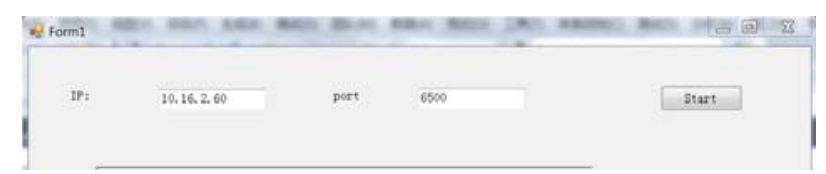

FIGURE IX. PARAMETER SETTING INTERFACE

The self-designed data collector is used to connect the GPRS with the computer. Power meter data being sent through the communication circuit to the collector, the collector sent it to the computer, using the computer's serial test software, transmitted to the specified database. Click on the start of the page query button, the meter data are sent, the experiment proved that the received data and the actual meter data consistent.

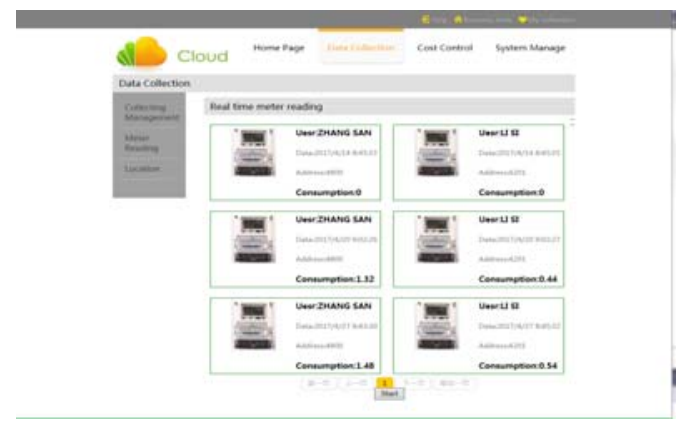

FIGURE X. HISTORICAL DATA INTERFACE

Comparing the last experimental data with the electric energy meter, the data are the same, which can prove that the system can transmit the data accurately and can be applied.

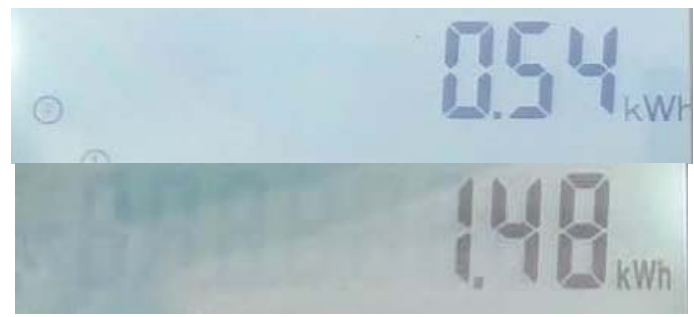

FIGURE XI. PHYSICAL DATA OF ELECTRIC ENERGY METER

\section{CONCLUSION}

The experiment shows that the data of the meter are consistent with the data collected and uploaded by the system, and the data can be copied accurately, and the system work is stable. The wireless remote intelligent meter designed by this system has the following advantages: it can read the meter data in real time and monitor the user's electricity use in real time. The installation and maintenance are convenient, the layout is simple, the working procedure of the wiring is eliminated and the cost is low. This system can solve the inconvenience of the meter reading of the management department, and it is the direction of the development of the intelligent meter.

\section{REFERENCES}

[1] Wu Xudong.. 2009. Design and implementation of electric meter monitoring system based on ZigBee Wireless Sensor Network Southwest Jiaotong University ,China.

[2] Li Dong, Di Juxing, Chen En. and Lin Tao. 2014. Design of a Si4432 based wireless ad hoc network meter reading system. Technical Report. University of Maryland at College Park.

[3] Zhang Mingda, and Huang Jianming. 2017. Design of wireless remote intelligent staircase billing water meter based on Si4432. Electronic measurement technology.(Jan,2017)

[4] Yu Ling. 2016. Design of distributed measurement system for industrial users based on WIA. Northeast China Electric Power University,Beijing.

[5] Zhao Hongke. 2014. Design of a distributed water meter wireless data acquisition system based on Si4432., Hebei University of Technology.

[6] Jiang Liming, and Zhuang Weidong. 2014. Research on the application of ZigBee/GPRS technology in precision agriculture. Journal of agricultural mechanization research 2014(04):179-182.

[7] Wang Yubin. 2016. A widget framework for augmented interaction in SCAPE. Electronic world. 2016.10.125. 